Claremont Colleges

Scholarship@ Claremont

All HMC Faculty Publications and Research

HMC Faculty Scholarship

$1-1-2003$

\title{
The Effect of the Domain Topology on the Number of Minimal Nodal Solutions of an Elliptic Equation at Critical Growth in a Symmetric Domain
}

\author{
Alfonso Castro \\ Harvey Mudd College \\ Mónica Clapp \\ Universidad Nacional Autonoma de Mexico
}

\section{Recommended Citation}

Castro, Alfonso and Monica Clapp. "The effect of the domain topology on the number of minimal nodal solutions of an elliptic equation at critical growth in a symmetric domain”, Nonlinearity 16(2003), 579-590.

This Article - postprint is brought to you for free and open access by the HMC Faculty Scholarship at Scholarship @ Claremont. It has been accepted for inclusion in All HMC Faculty Publications and Research by an authorized administrator of Scholarship @ Claremont. For more information, please contact scholarship@cuc.claremont.edu. 


\title{
The effect of the domain topology on the number of minimal nodal solutions of an elliptic equation at critical growth in a symmetric domain
}

\author{
Alfonso Castro $^{1}$ and Mónica Clapp ${ }^{2,3}$ \\ ${ }^{1}$ Department of Applied Mathematics, University of Texas at San Antonio, San Antonio, \\ TX 78249, USA \\ 2 Instituto de Matemáticas, Universidad Nacional Autónoma de México Circuito Exterior, \\ Ciudad Universitaria, 04510 México, D.F., Mexico \\ E-mail: castro@math.utsa.edu and mclapp@math.unam.mx
}

Received 9 September 2002, in final form 6 December 2002

Published 13 January 2003

Online at stacks.iop.org/Non/16/579

Recommended by F Merle

\begin{abstract}
We consider the Dirichlet problem $\Delta u+\lambda u+|u|^{2^{*}-2} u=0$ in $\Omega, u=0$ on $\partial \Omega$ where $\Omega$ is a bounded smooth domain in $\mathbb{R}^{N}, N \geqslant 4$, and $2^{*}=2 N /(N-2)$ is the critical Sobolev exponent. We show that if $\Omega$ is invariant under an orthogonal involution then, for $\lambda>0$ sufficiently small, there is an effect of the equivariant topology of $\Omega$ on the number of solutions which change sign exactly once.
\end{abstract}

Mathematics Subject Classification: 35J65, 35J20

\section{Introduction}

Consider the problem

$$
\left(\wp_{\lambda}\right) \begin{cases}\Delta u+\lambda u+|u|^{2^{*}-2} u=0 & \text { in } \Omega, \\ u=0 & \text { on } \partial \Omega,\end{cases}
$$

where $\Omega$ is a bounded smooth domain in $\mathbb{R}^{N}, N \geqslant 3,2^{*}=2 N /(N-2)$, and $\lambda \in \mathbb{R}$. We are interested in solutions to this problem which change sign exactly once, that is, solutions $u$ such that $\Omega \backslash u^{-1}(0)$ has exactly two connected components, $u$ is positive in one of them and negative in the other.

Let us first recall some well-known facts. If $\lambda=0$, this problem does not have a least energy solution, and it does not have a nontrivial solution, if $\Omega$ is strictly starshaped and $\lambda \leqslant 0$ [11]. In contrast with this situation, Brézis and Nirenberg [2] showed that there is at least one

3 Research partially supported by CONACYT, México, under grant 28031-E. 
positive solution of $\left(\wp_{\lambda}\right)$ if $N \geqslant 4$ and $0<\lambda<\lambda_{1}(\Omega)$, where $\lambda_{1}(\Omega)$ is the first eigenvalue of $-\Delta$ on $\Omega$ with boundary condition $u=0$. Furthermore, it was shown by Rey [12] for $N \geqslant 5$ and Lazzo [10] for $N \geqslant 4$ that there is an effect of the domain topology on the number of low energy positive solutions of this problem, namely, they showed that there is a $0<\bar{\lambda}<\lambda_{1}(\Omega)$ such that $\left(\wp_{\lambda}\right)$ has at least $\operatorname{cat}(\Omega)$ positive solutions for all $0<\lambda<\bar{\lambda}$, where cat $(\Omega)$ is the Lusternik-Schnirelmann category of $\Omega$.

The first result about sign changing solutions is due to Cerami et al [5] who showed the existence of a pair of least energy sign changing solutions if $N \geqslant 6$ and $0<\lambda<\lambda_{1}(\Omega)$. These solutions change sign exactly once. Similar results were obtained by Zhang [18] and Tarantello [16].

Some multiplicity results for sign changing solutions are also known. Cerami et al [5] showed the existence of infinitely many radially symmetric solutions on a ball for $N \geqslant 7$ and $0<\lambda<\lambda_{1}(\Omega)$. For domains with some special kind of symmetries, Fortunato and Jannelli [8] showed the existence of solutions with arbitrarily large energy for $N \geqslant 4$ and $\lambda>0$. However, these solutions change sign many times.

Here, we shall obtain a multiplicity result for solutions which change sign exactly once. We shall consider symmetric domains and prove that, as for positive solutions, there is also an effect of the domain topology on the number of such solutions. More precisely, we consider the problem

$$
\left(\wp_{\lambda}^{\tau}\right) \quad \begin{cases}\Delta u+\lambda u+|u|^{2^{*}-2} u=0 & \text { in } \Omega, \\ u=0 & \text { on } \partial \Omega, \\ u(\tau x)=-u(x) & \text { for all } x \in \Omega,\end{cases}
$$

where $\tau$ is a nontrivial orthogonal involution, that is, an orthogonal linear transformation of $\mathbb{R}^{N}$ such that $\tau \neq I$ and $\tau^{2}=I, I$ being the identity of $\mathbb{R}^{N}$, and $\Omega$ is a bounded smooth domain in $\mathbb{R}^{N}$ which is $\tau$-invariant, that is, $\tau x \in \Omega$ if $x \in \Omega$. This includes, e.g. domains $\Omega$ which are symmetric with respect to the origin (i.e. such that $x \in \Omega$ iff $-x \in \Omega$ ), as well as cylindrical or rotationally invariant domains as those considered by Fortunato and Jannelli. We shall prove the following.

Theorem 1. If $N \geqslant 4$ then, for every $0<\lambda<\lambda_{1}(\Omega)$, problem $\left(\wp_{\lambda}^{\tau}\right)$ has at least one pair of solutions which change sign exactly once.

Theorem 2. If $N \geqslant 4$ then there is a $0<\lambda^{*}<\lambda_{1}(\Omega)$ such that, for each $0<\lambda<\lambda^{*}$, problem $\left(\wp_{\lambda}^{\tau}\right)$ has at least $\tau-\operatorname{cat}_{\Omega}\left(\Omega \backslash \Omega^{\tau}\right)$ pairs of solutions which change sign exactly once.

Here, $\Omega^{\tau}=\{x \in \Omega: \tau x=x\}$ is the set of fixed points of the involution $\tau$, and $\tau$-cat is the $G_{\tau}$-equivariant Lusternik-Schnirelmann category for the group $G_{\tau}=\{I, \tau\}$. In many cases, the equivariant category turns out to be larger than the nonequivariant one. For example, for the unit sphere $\mathbb{S}^{N-1}$ in $\mathbb{R}^{N}$ and $\tau=-I, \tau$-cat $\left(\mathbb{S}^{N-1}\right)=N$ whereas cat $\left(\mathbb{S}^{N-1}\right)=2$. Thus, theorem 2 provides many solutions for some domains like the following.

Corollary 3. Let $\Omega$ be symmetric with respect to the origin and such that $0 \notin \Omega$. Assume further that there is an odd map $\varphi: \mathbb{S}^{N-1} \rightarrow \Omega$. Then if $N \geqslant 4$ there is a $0<\lambda^{*}<\lambda_{1}(\Omega)$ such that, for each $0<\lambda<\lambda^{*}$, problem $\left(\wp_{\lambda}\right)$ has at least $N$ pairs of odd solutions which change sign exactly once.

Theorem 1 is a special case of a result due to Hebey and Vaugon [9] who gave a condition for the existence of a least energy $\tau$-antisymmetric solution (i.e. a solution $u$ such that $u \circ \tau=-u$ ) for a larger class of problems. But theorem 2 says more: it says that for $\lambda$ small enough there is an effect of the $G_{\tau}$-equivariant topology of the domain on the number of $\tau$-antisymmetric solutions which change sign exactly once. 
The solutions provided by theorems 1 and 2 concentrate at symmetric points of the domain as $\lambda \rightarrow 0$. We shall show the following to hold.

Theorem 4. Let $N \geqslant 4$, let $\left(\lambda_{k}\right)$ be a sequence of positive numbers such that $\lambda_{k} \rightarrow 0$. The solutions $u_{k}$ to the problem $\left(\wp_{\lambda_{k}}^{\tau}\right)$ provided by theorems 1 and 2 satisfy the following: there is a sequence of points $\left(y_{k}\right)$ in $\Omega$ and a sequence of positive real numbers $\left(\varepsilon_{k}\right)$ such that

(i) $\left(\varepsilon_{k}\right)^{-1} \operatorname{dist}\left(y_{k}, \partial \Omega\right) \rightarrow \infty$ as $k \rightarrow \infty$,

(ii) $y_{k} \neq \tau y_{k}$ for all $k \in \mathbb{N}$, and $\varepsilon_{k}^{-1}\left|y_{k}-\tau y_{k}\right| \rightarrow \infty$ as $k \rightarrow \infty$,

(iii) $u_{k}=a_{N}\left[\left(\frac{\varepsilon_{k}}{\varepsilon_{k}^{2}+\left|x-y_{k}\right|^{2}}\right)^{(N-2) / 2}-\left(\frac{\varepsilon_{k}}{\varepsilon_{k}^{2}+\left|x-\tau y_{k}\right|^{2}}\right)^{(N-2) / 2}\right]+\mathrm{o}(1)$,

where $a_{N}=[N(N-2)]^{(N-2) / 4}$ and $\mathrm{o}(1) \rightarrow 0$ in $D^{1,2}\left(\mathbb{R}^{N}\right)$ as $k \rightarrow \infty$.

This paper is organized as follows. In section 2 , we describe the variational setting for problem $\left(\wp_{\lambda}^{\tau}\right)$. In section 3, we state a global compactness result for this problem, deduce some consequences of it, and prove theorem 1. Section 4 is devoted to the proof of theorems 2 and 4 . Finally, in section 5 we prove the global compactness result stated in section 3 which provides a precise description of all Palais-Smale sequences for the variational problem associated to $\left(\wp_{\lambda}^{\tau}\right)$.

\section{The variational problem}

We assume throughout that $0 \leqslant \lambda<\lambda_{1}(\Omega)$. We write $p=2^{*}=2 N /(N-2)$, and denote

$$
\|u\|_{\lambda}^{2}=\int_{\Omega}|\nabla u|^{2}-\lambda u^{2} \quad \text { and } \quad|u|_{p}^{p}=\int_{\Omega}|u|^{p} .
$$

Observe that, since $0 \leqslant \lambda<\lambda_{1}(\Omega),\|u\|_{\lambda}$ is a norm in the Sobolev space $H_{0}^{1}(\Omega)$ which is equivalent to the usual one.

The (classical) solutions of problem $\left(\wp_{\lambda}\right)$ are the critical points of the energy functional

$$
E_{\lambda}(u)=\frac{1}{2}\|u\|_{\lambda}^{2}-\frac{1}{p}|u|_{p}^{p}
$$

defined on $H_{0}^{1}(\Omega)$. The nontrivial critical points of $E_{\lambda}$ lie in the Nehari manifold

$$
\begin{aligned}
\mathcal{N}_{\lambda} & =\left\{u \in H_{0}^{1}(\Omega): u \neq 0, D E_{\lambda}(u) u=0\right\} \\
& =\left\{u \in H_{0}^{1}(\Omega): u \neq 0,\|u\|_{\lambda}^{2}=|u|_{p}^{p}\right\} .
\end{aligned}
$$

This is a manifold of class $C^{1}$ which is radially diffeomorphic to the unit sphere in $H_{0}^{1}(\Omega)$ [17, lemma 4.1].

The involution $\tau$ of $\Omega$ induces an orthogonal involution of $H_{0}^{1}(\Omega)$, which we also denote by $\tau$, as follows: for each $u \in H_{0}^{1}(\Omega)$ we define $\tau u \in H_{0}^{1}(\Omega)$ by

$$
(\tau u)(x)=-u(\tau x)
$$

The solutions of problem $\left(\wp_{\lambda}^{\tau}\right)$ are the critical points of $E_{\lambda}$ which lie in the closed linear subspace

$$
H_{0}^{1}(\Omega)^{\tau}=\left\{u \in H_{0}^{1}(\Omega): \tau u=u\right\}
$$

of $H_{0}^{1}(\Omega)$. Observe that $E_{\lambda}(\tau u)=E_{\lambda}(u)$ and that $\nabla E_{\lambda}(\tau u)=\tau \nabla E_{\lambda}(u)$. Thus $\tau \nabla E_{\lambda}(u)=$ $\nabla E_{\lambda}(u)$ if $\tau u=u$. Therefore, the nontrivial solutions of $\left(\wp_{\lambda}^{\tau}\right)$ are the critical points of the restriction of $E_{\lambda}$ to the $\tau$-invariant Nehari manifold

$$
\mathcal{N}_{\lambda}^{\tau}=\left\{u \in \mathcal{N}_{\lambda}: \tau u=u\right\}=\mathcal{N}_{\lambda} \cap H_{0}^{1}(\Omega)^{\tau} .
$$


Let

$$
\mu_{\lambda}=\mu_{\lambda}(\Omega)=\inf _{\mathcal{N}_{\lambda}} E_{\lambda} \quad \text { and } \quad \mu_{\lambda}^{\tau}=\mu_{\lambda}^{\tau}(\Omega)=\inf _{\mathcal{N}_{\lambda}^{\tau}} E_{\lambda} .
$$

If $\lambda=0$ then $\mu_{0}(\Omega)=(1 / N) S^{N / 2}$ where $S$ is the best Sobolev constant for the imbedding of $H_{0}^{1}(\Omega)$ into $L^{2^{*}}(\Omega)$. In particular, $\mu_{0}$ is independent of $\Omega$ and, due to the maximum principle, it is not achieved by $E_{0}$ on $\mathcal{N}_{0}$ if $\Omega \neq \mathbb{R}^{N}$. On the other hand, if $N \geqslant 4$, Brézis and Nirenberg [2] showed that, for $0<\lambda<\lambda_{1}(\Omega)$ and any bounded domain $\Omega$,

$$
\mu_{\lambda}(\Omega)<\mu_{0}=\frac{1}{N} S^{N / 2}
$$

and that $\mu_{\lambda}(\Omega)$ is achieved by $E_{\lambda}$ on $\mathcal{N}_{\lambda}$. Moreover,

$$
\lim _{\lambda \rightarrow 0} \mu_{\lambda}=\frac{1}{N} S^{N / 2}
$$

Proposition 5. Let $N \geqslant 4$. For every $0<\lambda<\lambda_{1}(\Omega)$, the following holds

$$
2 \mu_{\lambda} \leqslant \mu_{\lambda}^{\tau}<\mu_{0}^{\tau}=\frac{2}{N} S^{N / 2}
$$

Moreover, $\mu_{0}^{\tau}=(2 / N) S^{N / 2}$ is not achieved by $E_{0}$ on $\mathcal{N}_{0}^{\tau}$.

Proof. Let $u^{ \pm}= \pm \max \{ \pm u, 0\}$. Observe that, if $u=\tau u$, then $\left\|u^{+}\right\|_{\lambda}^{2}=\left\|u^{-}\right\|_{\lambda}^{2}$ and $\left|u^{+}\right|_{p}^{p}=\left|u^{-}\right|_{p}^{p}$. So, if $u \in \mathcal{N}_{\lambda}^{\tau}$ then $u^{+}, u^{-} \in \mathcal{N}_{\lambda}$ and

$$
E_{\lambda}(u)=E_{\lambda}\left(u^{+}\right)+E_{\lambda}\left(u^{-}\right) \geqslant 2 \mu_{\lambda} .
$$

This shows that $2 \mu_{\lambda} \leqslant \mu_{\lambda}^{\tau}$ for every $0 \leqslant \lambda<\lambda_{1}(\Omega)$. To prove the second inequality, choose $x \in \Omega$ with $\tau x \neq x$, and $r>0$ so that $B_{r}(x) \subset \Omega$ and $B_{r}(x) \cap B_{r}(\tau x)=\emptyset$, where $B_{r}(\xi)$ denotes the open ball in $\mathbb{R}^{N}$ with centre $\xi$ and radius $r$. For $0<\lambda<\lambda_{1}(\Omega)$, let $u_{\lambda, r}$ be the positive ground state solution of the problem

$$
\left(\wp_{\lambda, r}\right) \begin{cases}\Delta u+\lambda u+|u|^{2^{*}-2} u=0 & \text { in } B_{r}(0), \\ u=0 & \text { on } \partial B_{r}(0) .\end{cases}
$$

Then $\left\|u_{\lambda, r}\right\|_{\lambda}^{2}=\left|u_{\lambda, r}\right|_{p}^{p}$ and, since $u_{\lambda, r}$ is radially symmetric,

$$
u=u_{\lambda, r}(\cdot-x)-u_{\lambda, r}(\cdot-\tau x) \in \mathcal{N}_{\lambda}^{\tau} \text {. }
$$

Thus,

$$
\mu_{\lambda}^{\tau} \leqslant E_{\lambda}(u)=2 E_{\lambda}\left(u_{\lambda, r}\right)<\frac{2}{N} S^{N / 2} .
$$

To show that $\mu_{0}^{\tau} \leqslant(2 / N) S^{N / 2}$ we take a minimizing sequence for problem $\left(\wp_{0, r}\right)$ consisting of positive functions, i.e.

$$
u_{k} \in H_{0}^{1}\left(B_{r}(0)\right), \quad\left\|u_{k}\right\|^{2}=\left|u_{k}\right|_{p}^{p}, \quad u_{k}=u_{k}^{+}, \quad \lim _{k \rightarrow \infty} E_{0}\left(u_{k}\right)=\frac{1}{N} S^{N / 2} .
$$

Then, $v_{k}=u_{k}(\cdot-x)-u_{k}(\cdot+x) \in \mathcal{N}_{0}^{\tau}$ and

$$
\mu_{0}^{\tau} \leqslant \lim _{k \rightarrow \infty} E_{0}\left(v_{k}\right)=\lim _{k \rightarrow \infty} 2 E_{0}\left(u_{k}\right)=\frac{2}{N} S^{N / 2} .
$$

To prove the last assertion, we argue by contradiction. If $u \in \mathcal{N}_{0}^{\tau}$ were such that $E_{0}(u)=$ $(2 / N) S^{N / 2}$ then, since $u^{+}, u^{-} \in \mathcal{N}_{0}$ and $E_{0}(u)=E_{0}\left(u^{+}\right)+E_{0}\left(u^{-}\right)$, it would follow that $E_{0}\left(u^{+}\right)=E_{0}\left(u^{-}\right)=(1 / N) S^{N / 2}$. But $(1 / N) S^{N / 2}$ is not achieved by $E_{0}$ on $\mathcal{N}_{0}$. Therefore, $(2 / N) S^{N / 2}$ is not achieved by $E_{0}$ on $\mathcal{N}_{0}^{\tau}$.

Let $u$ be solution of problem $\left(\wp_{\lambda}\right)$. Then it is of class $C^{2}$. One says that $u$ changes sign $n$ times if the set $\{x \in \Omega: u(x) \neq 0\}$ has $n+1$ connected components. If $u$ is a solution of problem $\left(\wp_{\lambda}^{\tau}\right)$, then it changes sign an odd number of times. 
Proposition 6. If $u$ is a solution of problem $\left(\wp_{\lambda}^{\tau}\right)$ which changes sign $2 m-1$ times, then $E_{\lambda}(u) \geqslant m \mu_{\lambda}^{\tau}$.

Proof. The set $\{x \in \Omega: u(x)>0\}$ has $m$ connected components $A_{1}, \ldots, A_{m}$. Let $u_{i}(x)=u(x)$ if $x \in A_{i} \cup \tau A_{i}$ and $u_{i}(x)=0$, otherwise. Since $u$ is a critical point of $E_{\lambda}$,

$$
D E_{\lambda}(u) u_{i}=\int_{\Omega}\left(\nabla u \nabla u_{i}-\lambda u u_{i}-|u|^{p-2} u u_{i}\right)=\left\|u_{i}\right\|_{\lambda}^{2}-\left|u_{i}\right|_{p}^{p}=0 .
$$

Thus, $u_{i} \in \mathcal{N}_{\lambda}^{\tau}$ for all $i=1, \ldots, m$, and

$$
E_{\lambda}(u)=E_{\lambda}\left(u_{1}\right)+\cdots+E_{\lambda}\left(u_{m}\right) \geqslant m \mu_{\lambda}^{\tau} .
$$

\section{A compactness condition}

We recall that a sequence $\left(u_{k}\right)$ in $H_{0}^{1}(\Omega)$ such that

$$
E_{\lambda}\left(u_{k}\right) \rightarrow c, \quad\left\|D E_{\lambda}\left(u_{k}\right)\right\|_{H^{-1}(\Omega)} \rightarrow 0
$$

as $k \rightarrow \infty$ is called a Palais-Smale sequence (PS-sequence for short) for $E_{\lambda}$ at the level $c$. Struwe has given a complete description of all PS-sequences for $E_{\lambda}[13,14$, theorem 3.1]. For PS-sequences in $H_{0}^{1}(\Omega)^{\tau}$ a more precise description may be given as follows.

Theorem 7. Let $\left(u_{k}\right)$ be a PS-sequence for $E_{\lambda}$ such that $u_{k} \in H_{0}^{1}(\Omega)^{\tau}$. Then, after replacing $\left(u_{k}\right)$ by a subsequence if necessary, there exist a solution $u$ of problem $\left(\wp_{\lambda}^{\tau}\right)$, two numbers $m_{1}, m_{2} \geqslant 0$ and, for each $1 \leqslant i \leqslant m=m_{1}+m_{2}$, a sequence $\left(y_{i, k}\right)$ in $\Omega$, a sequence $\left(\varepsilon_{i, k}\right)$ in $(0, \infty)$, and a solution $\left(\tilde{u}_{i}\right)$ of the limiting problem

$$
\left(\wp_{\infty}\right) \quad \begin{cases}\Delta u+|u|^{2^{*}-2} u=0 & \text { in } \mathbb{R}^{N}, \\ u(x) \rightarrow 0 & \text { as }|x| \rightarrow 0,\end{cases}
$$

such that

(i) $\varepsilon_{i, k}^{-1} \operatorname{dist}\left(y_{i, k}, \partial \Omega\right) \rightarrow \infty$ as $k \rightarrow \infty$ for each $i=1, \ldots, m$,

(ii) $\tau y_{i, k} \neq y_{i, k}$ and $\varepsilon_{i, k}^{-1}\left|y_{i, k}-\tau y_{i, k}\right| \rightarrow \infty$ as $k \rightarrow \infty$ for each $i=1, \ldots, m_{1}$,

(iii) $\tau y_{i, k}=y_{i, k}$ and $\tau \tilde{u}_{i}=\tilde{u}_{i}$ for $i=m_{1}+1, \ldots, m$, for all $k \in \mathbb{N}$,

(iv) $u_{k}=u+\sum_{i=1}^{m_{1}} \varepsilon_{i, k}^{(2-N) / 2}\left[\tilde{u}_{i}\left(\frac{\cdot-y_{i, k}}{\varepsilon_{i, k}}\right)+\left(\tau \tilde{u}_{i}\right)\left(\frac{\cdot-\tau y_{i, k}}{\varepsilon_{i, k}}\right)\right]$

$$
+\sum_{i=m_{1}+1}^{m} \varepsilon_{i, k}^{(2-N) / 2} \tilde{u}_{i}\left(\frac{-y_{i, k}}{\varepsilon_{i, k}}\right)+\mathrm{o}(1),
$$

where $\mathrm{o}(1) \rightarrow 0$ in $D^{1,2}\left(\mathbb{R}^{N}\right)$ as $k \rightarrow \infty$,

(v) $E_{\lambda}\left(u_{k}\right) \rightarrow E_{\lambda}(u)+2 \sum_{i=1}^{m_{1}} E_{0}\left(\tilde{u}_{i}\right)+\sum_{i=m_{1}+1}^{m} E_{0}\left(\tilde{u}_{i}\right)$ as $k \rightarrow \infty$.

The proof goes along the lines of [3, theorem 1]. We postpone it to section 5 . Now we point out some consequences which are relevant to our purposes.

We say that $E_{\lambda}$ satisfies the $\tau$-PS condition (PS) ${ }_{c}^{\tau}$ at the level $c$ if every sequence $\left(u_{k}\right)$ such that

$u_{k} \in H_{0}^{1}(\Omega)^{\tau}, \quad E_{\lambda}\left(u_{k}\right) \rightarrow c \quad$ and $\quad\left\|D E_{\lambda}\left(u_{k}\right)\right\|_{H^{-1}(\Omega)} \rightarrow 0 \quad$ as $k \rightarrow \infty$,

has a convergent subsequence. An immediate consequence of theorem 7 is the following.

Corollary 8. $E_{\lambda}$ satisfies $(P S)_{c}^{\tau}$ at every $c<(2 / N) S^{N / 2}$. 
Proof. If $\tilde{u}$ is a nontrivial solution of problem $\left(\wp_{\infty}\right)$ then $E_{0}(\tilde{u}) \geqslant(1 / N) S^{N / 2}$. If, furthermore, $\tau \tilde{u}=\tilde{u}$ then $E_{0}(\tilde{u}) \geqslant(2 / N) S^{N / 2}$. So, if $\left(u_{k}\right)$ is a PS-sequence at the level $c$ such that $u_{k} \in H_{0}^{1}(\Omega)^{\tau}$, both numbers $m_{1}$ and $m_{2}$ provided by theorem 7 must be cero. Hence, up to a subsequence, $u_{k} \rightarrow u$.

Recall that, up to sign, the nontrivial least energy solutions of the limiting problem $\left(\wp_{\infty}\right)$ are the instantons

$U_{\varepsilon, z}(x)=a_{N}\left(\frac{\varepsilon}{\varepsilon^{2}+|x-z|^{2}}\right)^{(N-2) / 2}, \quad a_{N}=[N(N-2)]^{(N-2) / 4}, \quad \varepsilon>0, \quad z \in \mathbb{R}^{N}$,

cf $[1,15]$. They satisfy

$$
\int_{\mathbb{R}^{N}}\left|\nabla U_{\varepsilon, z}\right|^{2}=S^{N / 2}=\int_{\mathbb{R}^{N}}\left|U_{\varepsilon, z}\right|^{2^{*}}
$$

Another consequence of theorem 7 is the following.

Corollary 9. If $\left(u_{k}\right)$ is a PS-sequence for $E_{0}$ in $H_{0}^{1}(\Omega)^{\tau}$ such that $E_{0}\left(u_{k}\right) \rightarrow(2 / N) S^{N / 2}$ then there is a sequence of points $\left(y_{k}\right)$ in $\Omega$ and a sequence of positive real numbers $\left(\varepsilon_{k}\right)$ such that

(i) $\left(\varepsilon_{k}\right)^{-1} \operatorname{dist}\left(y_{k}, \partial \Omega\right) \rightarrow \infty$ as $k \rightarrow \infty$,

(ii) $y_{k} \neq \tau y_{k}$ for all $k \in N$, and $\varepsilon_{k}^{-1}\left|y_{k}-\tau y_{k}\right| \rightarrow \infty$ as $k \rightarrow \infty$,

(iii) $\left\|u_{k}-U_{\varepsilon_{k}, y_{k}}+U_{\varepsilon_{k}, \tau y_{k}}\right\| \rightarrow 0$ in $D^{1,2}\left(\mathbb{R}^{N}\right)$ as $k \rightarrow \infty$.

Proof. If there were a solution $\tilde{u}$ of $\left(\wp_{\infty}\right)$ such that $\tau \tilde{u}=\tilde{u}$ and $E_{0}(\tilde{u})=(2 / N) S^{N / 2}$ then

$$
\int_{\mathbb{R}^{N}}\left|\nabla \tilde{u}^{ \pm}\right|^{2}=S^{N / 2}=\int_{\mathbb{R}^{N}}\left|\tilde{u}^{ \pm}\right|^{2^{*}}
$$

Thus, $\tilde{u}^{+}$and $\tilde{u}^{-}$would be solutions of $\left(\wp_{\infty}\right)$ which vanish in some open subset of $\mathbb{R}^{N}$. This is a contradiction. Therefore, every solution $\tilde{u}$ of $\left(\wp_{\infty}\right)$ such that $\tau \tilde{u}=\tilde{u}$ must satisfy $E_{0}(\tilde{u})>(2 / N) S^{N / 2}$. On the other hand, we have shown (proposition 5) that problem $\left(\wp_{0}^{\tau}\right)$ does not have a nontrivial least energy solution in $\Omega$. So theorem 7 implies the result.

Proof of theorem 1. Take a minimizing sequence $\left(u_{k}\right)$ for $E_{\lambda}$ on $\mathcal{N}_{\lambda}^{\tau}$. By Ekeland's variational principle [7, 17, theorem 8.5], we may assume that it is a PS-sequence. Proposition 5 and corollary 8 yield the existence of a minimum of $E_{\lambda}$ on $\mathcal{N}_{\lambda}^{\tau}$, and proposition 6 asserts that it changes sign exactly once.

\section{The effect of the domain}

We recall some facts about equivariant Lusternik-Schnirelmann theory. If $G$ is a compact Lie group, then a $G$-space is a topological space $X$ with a continuous $G$-action $G \times X \rightarrow X$, $(g, x) \mapsto g x$. A $G$-map is a continuous function $f: X \rightarrow Y$ between $G$-spaces $X$ and $Y$ which is compatible with the $G$-actions, i.e. $f(g x)=g f(x)$ for all $x \in X, g \in G$. Two $G$-maps $f_{0}, f_{1}: X \rightarrow Y$ are $G$-homotopic if there is a homotopy $\Theta: X \times[0,1] \rightarrow Y$ such that $\Theta(x, 0)=f_{0}(x), \Theta(x, 1)=f_{1}(x)$ and $\Theta(g x, t)=g \Theta(x, t)$ for all $x \in X, g \in G$, $t \in[0,1]$. A subset $A$ of a $X$ is $G$-invariant if $g a \in A$ for every $a \in A, g \in G$. The $G$-orbit of a point $x \in X$ is the set $G x=\{g x: g \in G\}$. A detailed discussion on $G$-spaces may be found, e.g. in [6].

In our applications, $G$ will be the group with two elements, acting as $G_{\tau}=\{I, \tau\}$ on $\Omega$, and as $\mathbb{Z} / 2=\{1,-1\}$ by multiplication on the Nehari manifold $\mathcal{N}_{\lambda}^{\tau}$. The energy functional $E_{\lambda}: \mathcal{N}_{\lambda}^{\tau} \rightarrow \mathbb{R}$ is a $\mathbb{Z} / 2$-map for this action, in other words, it is an even functional. 
Definition 10. The $G$-category of a $G$-map $f: X \rightarrow Y$ is the smallest number $k=G$-cat $(f)$ of open $G$-invariant subsets $X_{1}, \ldots, X_{k}$ of $X$ which cover $X$ and which have the property that, for each $i=1, \ldots, k$, there is a point $y_{i} \in Y$ and $a G$-map $\alpha_{i}: X_{i} \rightarrow G y_{i} \subset Y$ such that the restriction of $f$ to $X_{i}$ is $G$-homotopic to $\alpha_{i}$. If no such covering exists we define $G$-cat $(f)=\infty$.

If $A$ is a $G$-subset of $X$ and $\iota: A \hookrightarrow X$ is the inclusion map we write

$$
G \text {-cat }{ }_{X}(A)=G \text {-cat }(\iota) \quad \text { and } \quad G \text {-cat }(X)=G \text {-cat } X(X) .
$$

The following properties can be easily verified.

\section{Lemma 11.}

(a) If $f: X \rightarrow Y$ and $h: Y \rightarrow Z$ are $G$-maps then

$$
G \text {-cat }(h \circ f) \leqslant \min \{G \text {-cat }(f), G \text {-cat }(h)\} .
$$

In particular, $G$-cat $(h \circ f) \leqslant G$-cat $(Y)$.

(b) If $f_{0}, f_{1}: X \rightarrow Y$ are $G$-homotopic then $G$-cat $\left(f_{0}\right)=G$-cat $\left(f_{1}\right)$.

Equivariant Lusternik-Schnirelmann category provides a lower bound for the number of critical $G$-orbits of a $G$-invariant functional. The following result is well known (see, e.g. [4, theorem $1.1 ; 14$, theorem 5.7]).

Theorem 12. Let $\phi: M \rightarrow \mathbb{R}$ be an even $C^{1}$-functional on a complete $C^{1,1}$-submanifold $M$ of a Banach space which is symmetric with respect to the origin. Assume that $\phi$ is bounded below and satisfies the PS condition $(P S)_{c}$ for every $c \leqslant d$. Then $\phi$ has at least $\mathbb{Z} / 2$-cat $\left(\phi^{d}\right)$ antipodal pairs $\{u,-u\}$ of critical points with critical values $\phi( \pm u) \leqslant d$.

Here, $\phi^{d}$ stands, as usual, for the sublevel set

$$
\phi^{d}=\{u \in M: \phi(u) \leqslant d\}
$$

and the group $\mathbb{Z} / 2=\{1,-1\}$ acts by multiplication on $V$. There is a similar result for arbitrary group actions [4, theorem 1.1].

Coming back to our problem, we assume from now on that $N \geqslant 4$ and $0<\lambda<\lambda_{1}(\Omega)$. Given $r>0$ let

$$
\begin{aligned}
& \Omega_{r}^{-}=\left\{x \in \Omega: \operatorname{dist}\left(x, \partial \Omega \cup \Omega^{\tau}\right) \geqslant r\right\}, \\
& \Omega_{r}^{+}=\left\{x \in \mathbb{R}^{N}: \operatorname{dist}(x, \Omega) \leqslant r\right\},
\end{aligned}
$$

where $\Omega^{\tau}=\{x \in \Omega: \tau x=x\}$. Fix $r>0$ such that the inclusion maps $\Omega_{r}^{-} \hookrightarrow \Omega \backslash \Omega^{\tau}$ and $\Omega \hookrightarrow \Omega_{r}^{+}$are $G_{\tau}$-homotopy equivalences where $G_{\tau}=\{I, \tau\}$. We shall start by proving the following.

Proposition 13. Let $N \geqslant 4$. There is a $0<\lambda^{*}<\lambda_{1}(\Omega)$ and, for each $0<\lambda<\lambda^{*}$, there is a $\mu_{\lambda}^{\tau}<d_{\lambda}<(2 / N) S^{N / 2}$ and two maps

$$
\Omega_{r}^{-} \stackrel{\alpha_{\lambda}}{\longrightarrow} \mathcal{N}_{\lambda}^{\tau} \cap E_{\lambda}^{d_{\lambda}} \stackrel{\beta_{\lambda}}{\longrightarrow} \Omega_{r}^{+}
$$

such that $\alpha_{\lambda}(\tau x)=-\alpha_{\lambda}(x), \beta_{\lambda}(-u)=\tau \beta_{\lambda}(u)$, and $\beta_{\lambda} \circ \alpha_{\lambda}$ is $G_{\tau}$-homotopic to the inclusion $\operatorname{map} \Omega_{r}^{-} \hookrightarrow \Omega_{r}^{+}$.

For the proof we need the following lemmas. Consider the baricenter map $\beta: H_{0}^{1}(\Omega) \backslash\{0\} \rightarrow \mathbb{R}^{N}$,

$$
\beta(u)=\frac{\int_{\mathbb{R}^{N}}|u(x)|^{p} x \mathrm{~d} x}{\int_{\mathbb{R}^{N}}|u(x)|^{p} \mathrm{~d} x} .
$$


Lemma 14. Given $r>0$ there exists $\kappa>0$ such that, if $u \in \mathcal{N}_{0}^{\tau}$ and $E_{0}(u) \leqslant$ $(2 / N) S^{N / 2}+2 \kappa$, then

$$
\beta\left(u^{+}\right) \in \Omega_{r}^{+} .
$$

Proof. We argue by contradiction. Assume that for every $k \in \mathbb{N}$ there is a $u_{k} \in \mathcal{N}_{0}^{\tau}$ such that $\beta\left(u_{k}^{+}\right) \notin \Omega_{r}^{+}$and $E_{0}\left(u_{k}\right) \leqslant(2 / N) S^{N / 2}+(1 / 2 k)$. Then $\left(u_{k}^{+}\right)$is a minimizing sequence for $E_{0}$ in $\mathcal{N}_{0}$. By Ekeland's variational principle $[7,17$, theorem 8.5$]$ we may assume that $\left(u_{k}^{+}\right)$is a PS-sequence. Thus, by Struwe's theorem [13,14, theorem 3.1], there are sequences $\left(y_{k}\right)$ in $\Omega$ and $\left(\varepsilon_{k}\right)$ in $(0, \infty)$ such that

$$
\left\|u_{k}^{+}-U_{\varepsilon_{k}, y_{k}}\right\| \rightarrow 0 \quad \text { in } D^{1,2}\left(\mathbb{R}^{N}\right) \quad \text { as } k \rightarrow \infty .
$$

Therefore,

$$
\left|\beta\left(u_{k}^{+}\right)-\beta\left(U_{\varepsilon_{k}, y_{k}}\right)\right|=\left|\beta\left(u_{k}^{+}\right)-y_{k}\right| \rightarrow 0 \quad \text { as } k \rightarrow \infty,
$$

contradicting our assumption that $\beta\left(u_{k}^{+}\right) \notin \Omega_{r}^{+}$.

Let $\rho_{\lambda}: H_{0}^{1}(\Omega) \backslash\{0\} \rightarrow \mathcal{N}_{\lambda}$ be the radial projection onto the Nehari manifold, i.e.

$$
\rho_{\lambda}(u)=\frac{\|u\|_{\lambda}^{(N-2) / 2}}{|u|_{p}^{N / 2}} u .
$$

Lemma 15. For every $\kappa>0$ there is a $0<\lambda^{*}<\lambda_{1}(\Omega)$ such that, for each $0<\lambda<\lambda^{*}$, the following holds:

(a) If $u \in \mathcal{N}_{\lambda}^{\tau}$ is such that $E_{\lambda}(u) \leqslant \mu_{\lambda}^{\tau}+\kappa$ then $\rho_{0}(u) \in \mathcal{N}_{0}^{\tau}$ is such that $E_{0}\left(\rho_{0}(u)\right) \leqslant$ $(2 / N) S^{N / 2}+2 \kappa$.

(b) $(2 / N) S^{N / 2} \leqslant \mu_{\lambda}^{\tau}+\kappa$.

\section{Proof.}

(a) If $u \in \mathcal{N}_{\lambda}$ then

$$
\rho_{0}(u)=\frac{\|u\|^{(N-2) / 2}}{|u|_{p}^{N / 2}} u=\left(\frac{\|u\|}{\|u\|_{\lambda}}\right)^{(N-2) / 2} u .
$$

Let $\theta>0$ be such that $(1+\theta)^{N / 2}\left((2 / N) S^{N / 2}+\kappa\right) \leqslant(2 / N) S^{N / 2}+2 \kappa$. Then, if $\lambda \leqslant(\theta /(1+\theta)) \lambda_{1}(\Omega)$ and $E_{\lambda}(u) \leqslant \mu_{\lambda}^{\tau}+\kappa$,

$$
\begin{aligned}
E_{0}\left(\rho_{0}(u)\right) & =\frac{1}{N}\left(\frac{\|u\|}{\|u\|_{\lambda}}\right)^{N-2}\|u\|^{2} \\
& =\frac{1}{N}\left(\frac{\|u\|}{\|u\|_{\lambda}}\right)^{N}\|u\|_{\lambda}^{2} \\
& \leqslant\left(\frac{\lambda_{1}(\Omega)}{\lambda_{1}(\Omega)-\lambda}\right)^{N / 2} \frac{1}{N}\|u\|_{\lambda}^{2} \\
& \leqslant(1+\theta)^{N / 2} E_{\lambda}(u) \\
& \leqslant \frac{2}{N} S^{N / 2}+2 \kappa .
\end{aligned}
$$

(b) By proposition 5, $2 \mu_{\lambda} \leqslant \mu_{\lambda}^{\tau}<(2 / N) S^{N / 2}$. Since $\mu_{\lambda} \rightarrow(1 / N) S^{N / 2}$ as $\lambda \rightarrow 0$ there exists $0<\lambda^{*} \leqslant(\theta /(1+\theta)) \lambda_{1}(\Omega)$ such that

$$
\frac{2}{N} S^{N / 2}-\mu_{\lambda}^{\tau}<\kappa
$$

if $0<\lambda<\lambda^{*}$. 
We are ready to prove proposition 13 .

Proof of proposition 13. For $\kappa>0$ as in lemma 14 choose $\lambda^{*}$ as in proposition 15. Fix $0<\lambda<\lambda^{*}$ and let $u_{\lambda, r}$ be the positive ground state solution of the problem

$$
\left(\wp_{\lambda, r}\right) \begin{cases}\Delta u+\lambda u+|u|^{2^{*}-2} u=0 & \text { in } B_{r}(0), \\ u=0 & \text { on } \partial B_{r}(0) .\end{cases}
$$

Choose $d_{\lambda}$ such that $2 E_{\lambda}\left(u_{\lambda, r}\right)<d_{\lambda}<(2 / N) S^{N / 2}$ and define

$$
\begin{array}{ll}
\alpha_{\lambda}: \Omega_{r}^{-} \rightarrow \mathcal{N}_{\lambda}^{\tau} \cap E_{\lambda}^{d_{\lambda}}, & \alpha_{\lambda}(x)=u_{\lambda, r}(\cdot-x)-u_{\lambda, r}(\cdot-\tau x), \\
\beta_{\lambda}: \mathcal{N}_{\lambda}^{\tau} \cap E_{\lambda}^{d_{\lambda}} \rightarrow \Omega_{r}^{+}, & \beta_{\lambda}(u)=\beta\left(\rho_{0}(u)^{+}\right)=\beta\left(u^{+}\right) .
\end{array}
$$

These maps have obviously the desired properties.

Proof of theorem 2. Let $0<\lambda^{*}<\lambda_{1}(\Omega)$ and, for $0<\lambda<\lambda^{*}$, let $\mu_{\lambda}^{\tau}<d_{\lambda}<(2 / N) S^{(N / 2)}$ be as in proposition 13. Since $E_{\lambda}: \mathcal{N}_{\lambda}^{\tau} \rightarrow \mathbb{R}$ is even, bounded below and satisfies (PS) ${ }_{c}^{\tau}$ for $c<(2 / N) S^{N / 2}$ it follows from theorem 12 that $E_{\lambda}$ has at least $\mathbb{Z} / 2$-cat $\left(\mathcal{N}_{\lambda}^{\tau} \cap E_{\lambda}^{d_{\lambda}}\right)$ pairs $\pm u$ of critical points in $\mathcal{N}_{\lambda}^{\tau}$ with $E_{\lambda}( \pm u) \leqslant d_{\lambda}$ where $\mathbb{Z} / 2=\{1,-1\}$ acts by multiplication on $\mathcal{N}_{\lambda}^{\tau} \cap E_{\lambda}^{d_{\lambda}}$. On the other hand, lemma 11 and proposition 13 imply that

$G_{\tau}-\operatorname{cat}_{\Omega}\left(\Omega \backslash \Omega^{\tau}\right)=G_{\tau}-\operatorname{cat}\left(\Omega_{r}^{-} \hookrightarrow \Omega_{r}^{+}\right)=G_{\tau}-\operatorname{cat}\left(\beta_{\lambda} \circ \alpha_{\lambda}\right) \leqslant \mathbb{Z} / 2-\operatorname{cat}\left(\mathcal{N}_{\lambda}^{\tau} \cap E_{\lambda}^{d_{\lambda}}\right)$.

Taking $\lambda^{*}$ even smaller if necessary we may assume that $d_{\lambda}<2 \mu_{\lambda}^{\tau}$. Thus, by proposition 6 , these solutions change sign exactly once.

Proof of corollary 3. If $\{I,-I\}-\operatorname{cat}(\Omega)=k$, then there exists an odd map $\Omega \rightarrow \mathbb{S}^{k-1}$. Indeed: given an open covering $\left\{X_{1}, \ldots, X_{k}\right\}$ of $\Omega$ and odd maps $\alpha_{i}: X_{i} \rightarrow\left\{e_{i},-e_{i}\right\}$, where $\left\{e_{1}, \ldots, e_{k}\right\}$ is the canonical orthonormal basis of $\mathbb{R}^{k}$, let $\left\{\pi_{i}: X_{i} \rightarrow[0,1]\right\}$ be a partition of unity subordinated to the covering consisting of even functions. Then

$$
\psi(x)=\frac{\sum_{i=1}^{k} \pi_{i}(x) \alpha_{i}(x)}{\left\|\sum_{i=1}^{k} \pi_{i}(x) \alpha_{i}(x)\right\|}
$$

defines an odd map $\psi: \Omega \rightarrow \mathbb{S}^{k-1}$. Composing it with $\varphi$ gives an odd map $\psi \circ \varphi$ : $\mathbb{S}^{N-1} \rightarrow \mathbb{S}^{k-1}$ and the Borsuk-Ulam theorem implies that $N \geqslant k$. The result now follows from theorem 2 .

Proof of theorem 4. The solutions $u_{k}$ to problem $\left(\wp_{\lambda_{k}}^{\tau}\right)$ provided by theorems 1 and 2 satisfy $u_{k} \in \mathcal{N}_{\lambda_{k}}^{\tau}$ and $\mu_{\lambda_{k}}^{\tau} \leqslant E_{\lambda_{k}}\left(u_{k}\right)<(2 / N) S^{N / 2}$. As in lemma 15,

$$
\frac{2}{N} S^{N / 2} \leqslant E_{0}\left(\rho_{0}\left(u_{k}\right)\right) \leqslant\left(\frac{\lambda_{1}(\Omega)}{\lambda_{1}(\Omega)-\lambda_{k}}\right)^{N / 2} E_{\lambda_{k}}\left(u_{k}\right) \rightarrow \frac{2}{N} S^{N / 2}
$$

as $k \rightarrow \infty$, where $\rho_{0}$ is the radial projection onto $\mathcal{N}_{0}$. By corollary 9 the sequence $\left(\rho_{0}\left(u_{k}\right)\right)$ has the desired form. On the other hand,

$$
\left\|\rho_{0}\left(u_{k}\right)-u_{k}\right\|=\left|\left(\frac{\left\|u_{k}\right\|}{\left\|u_{k}\right\|_{\lambda_{k}}}\right)^{(N-2) / 2}-1\right|\left\|u_{k}\right\| \rightarrow 0
$$

as $k \rightarrow \infty$. 


\section{Proof of theorem 7}

As in Struwe's global compactness result $[13,14,17]$, theorem 7 follows inductively from the following proposition (cf [3]).

Proposition 16. Let $\left(u_{k}\right)$ be a PS-sequence for $E_{0}$ such that $u_{k} \in H_{0}^{1}(\Omega)^{\tau}, u_{k} \rightarrow 0$ weakly in $H_{0}^{1}(\Omega)^{\tau}$ and $E_{0}\left(u_{k}\right) \rightarrow c>0$. Then, replacing $\left(u_{k}\right)$ by a subsequence if necessary, there exist a sequence $\left(y_{k}\right)$ in $\Omega$, a sequence $\varepsilon_{k}$ in $(0, \infty)$, a solution $\tilde{u}$ of the limiting problem

$$
(\wp \infty) \begin{cases}\Delta u+|u|^{2^{*}-2} u=0 & \text { in } \mathbb{R}^{N}, \\ u(x) \rightarrow 0 & \text { as }|x| \rightarrow 0\end{cases}
$$

and a PS-sequence $\left(v_{k}\right)$ for $E_{0}$ such that $v_{k} \in H_{0}^{1}(\Omega)^{\tau}, \varepsilon_{k}^{-1} \operatorname{dist}\left(y_{k}, \partial \Omega\right) \rightarrow \infty$ as $k \rightarrow \infty$ and one of the following two assertions holds:

$$
\begin{aligned}
& \text { (I) } y_{k} \neq \tau y_{k}, \varepsilon_{k}^{-1}\left|y_{k}-\tau y_{k}\right| \rightarrow \infty \text { as } k \rightarrow \infty, \\
& u_{k}=v_{k}+\varepsilon_{k}^{(2-N) / 2}\left[\tilde{u}\left(\frac{-y_{k}}{\varepsilon_{k}}\right)-\tilde{u}\left(\tau\left(\frac{-\tau y_{k}}{\varepsilon_{k}}\right)\right)\right]+o(1) \text { in } D^{1,2}\left(\mathbb{R}^{N}\right), \\
& \quad \text { and } E_{0}\left(v_{k}\right) \rightarrow c-2 E_{0}(\tilde{u}) \text { as } k \rightarrow \infty . \\
& \text { (II) } y_{k}=\tau y_{k}, \tilde{u} \circ \tau=-\tilde{u}, \\
& \quad u_{k}=v_{k}+\varepsilon_{k}^{(2-N) / 2} \tilde{u}\left(\frac{-y_{k}}{\varepsilon_{k}}\right)+o(1) \quad \text { in } D^{1,2}\left(\mathbb{R}^{N}\right), \\
& \text { and } E_{0}\left(v_{k}\right) \rightarrow c-E_{0}(\tilde{u}) \text { as } k \rightarrow \infty .
\end{aligned}
$$

Proof. The proof will follow in several steps.

(1) Since PS-sequences for $E_{0}$ are bounded in $H_{0}^{1}(\Omega)$,

$$
\int_{\Omega}\left|u_{k}\right|^{2^{*}} \mathrm{~d} x=N E_{0}\left(u_{k}\right)-\frac{N}{2} D E_{0}\left(u_{k}\right) u_{k} \rightarrow N c>0 .
$$

Let $\delta=\min \left\{N c / 2,(S / 2)^{N / 2}\right\}$ where $S$ is the best Sobolev constant for the embedding of $H_{0}^{1}(\Omega)$ in $L^{2^{*}}(\Omega)$. Let $B(x, r)$ denote the closed ball in $\mathbb{R}^{N}$ with centre $x$ and radius $r$. The Levy concentration function

$$
\Phi_{k}(r):=\sup _{x \in \mathbb{R}^{N}} \int_{B(x, r)}\left|u_{k}\right|^{2^{*}}
$$

satisfies that $\Phi_{k}(0)=0$ and $\Phi_{k}(\infty)>\delta$ for $k$ large enough. Hence, we may choose $\xi_{k} \in \Omega$ and $\varepsilon_{k}>0$ such that

$$
\sup _{x \in \mathbb{R}^{N}} \int_{B\left(x, \varepsilon_{k}\right)}\left|u_{k}\right|^{2^{*}}=\int_{B\left(\xi_{k}, \varepsilon_{k}\right)}\left|u_{k}\right|^{2^{*}}=\delta .
$$

Observe that, since $\Omega$ is bounded, the sequence $\left(\varepsilon_{k}\right)$ is bounded.

(2) Let $\xi_{k}^{\tau}$ be the orthogonal projection of $\xi_{k}$ onto the fixed point set $\left\{x \in \mathbb{R}^{N}: \tau x=x\right\}$. We distinguish two cases and define $y_{k}$ as follows:

(I) $y_{k}=\xi_{k}$ if $\left(\varepsilon_{k}^{-1}\left|\xi_{k}-\xi_{k}^{\tau}\right|\right)$ is unbounded,

(II) $y_{k}=\xi_{k}^{\tau}$ if $\left(\varepsilon_{k}^{-1}\left|\xi_{k}-\xi_{k}^{\tau}\right|\right)$ is bounded.

Taking a subsequence if necessary we may assume that, in case $(I), y_{k} \neq \tau y_{k}$ for all $k$. Let $\tilde{u}_{k} \in D^{1,2}\left(\mathbb{R}^{N}\right)$ be given by

$$
\tilde{u}_{k}(z):=\varepsilon_{k}^{(N-2) / 2} u_{k}\left(\varepsilon_{k} z+y_{k}\right) .
$$


Notice that in case $(I I) \tilde{u}_{k} \circ \tau=-\tilde{u}_{k}$. Since

$$
\int\left|\nabla \tilde{u}_{k}\right|^{2}=\int\left|\nabla u_{k}\right|^{2} \quad \text { and } \quad \int\left|\tilde{u}_{k}\right|^{2^{*}}=\int\left|u_{k}\right|^{2^{*}},
$$

up to a subsequence, $\tilde{u}_{k} \rightarrow \tilde{u}$ weakly in $D^{1,2}\left(\mathbb{R}^{N}\right), \tilde{u}_{k} \rightarrow \tilde{u}$ a.e. on $\mathbb{R}^{N}$ and $\tilde{u}_{k} \rightarrow \tilde{u}$ in $L_{\text {loc }}^{2}\left(\mathbb{R}^{N}\right)$. If $\tilde{u} \equiv 0$ then, for every $z \in \mathbb{R}^{N}$ and every $h \in C_{c}^{\infty}(B(z, 1))$,

$$
\begin{aligned}
S\left(\int\left|h \tilde{u}_{k}\right|^{2^{*}}\right)^{2 / 2^{*}} & \leqslant \int\left|\nabla\left(h \tilde{u}_{k}\right)\right|^{2}=\int \nabla \tilde{u}_{k} \cdot \nabla\left(h^{2} \tilde{u}_{k}\right)+\int|\nabla h|^{2} \tilde{u}_{k}^{2} \\
& =\int h^{2}\left|\tilde{u}_{k}\right|^{2^{*}}-D E_{0}\left(u_{k}\right)\left(\left[h^{2}\left(\frac{\cdot-y_{k}}{\varepsilon_{k}}\right)\right] u_{k}\right)+o(1) \\
& \leqslant\left(\int\left|\tilde{u}_{k}\right|^{2^{*}}\right)^{2 / N}\left(\int\left|h \tilde{u}_{k}\right|^{2^{*}}\right)^{2 / 2^{*}}+o(1) \\
& \leqslant \delta^{2 / N}\left(\int\left|h \tilde{u}_{k}\right|^{2^{*}}\right)^{2 / 2^{*}}+o(1) \\
& \leqslant \frac{S}{2}\left(\int\left|h \tilde{u}_{k}\right|^{2^{*}}\right)^{2 / 2^{*}}+o(1),
\end{aligned}
$$

where the first inequality is Sobolev's inequality, the second one follows from the fact that $\left(u_{k}\right)$ is a PS-sequence and from Hölder's inequality, the third one uses $(*)$ and the fourth one our definition of $\delta$. It follows that $\tilde{u}_{k} \rightarrow 0$ in $L_{\text {loc }}^{2^{*}}\left(\mathbb{R}^{N}\right)$. On the other hand, since $\varepsilon_{k}^{-1}\left|\xi_{k}-y_{k}\right|<C<\infty$ for all $k$

$$
0<\delta=\int_{B\left(\xi_{k}, \varepsilon_{k}\right)}\left|u_{k}\right|^{2^{*}} \leqslant \int_{B\left(y_{k}, \varepsilon_{k}(C+1)\right)}\left|u_{k}\right|^{2^{*}}=\int_{B(0, C+1)}\left|\tilde{u}_{k}\right|^{2^{*}} .
$$

This is a contradiction. Therefore, $\tilde{u} \not \equiv 0$.

(3) Since $\Omega$ is bounded and $u_{k} \rightarrow 0$ weakly in $H_{0}^{1}(\Omega)$, up to a subsequence, $y_{k} \rightarrow y \in \bar{\Omega}$ and $\varepsilon_{k} \rightarrow 0$.

If $\left(\varepsilon_{k}^{-1} \operatorname{dist}\left(y_{k}, \partial \Omega\right)\right)$ were bounded then $\tilde{u}$ would be a solution of $-\Delta u=|u|^{2^{*}-2} u$ in a half space and, by Pohozaev's identity [11], $\tilde{u} \equiv 0$. This is a contradiction. Therefore,

$$
\varepsilon_{k}^{-1} \operatorname{dist}\left(y_{k}, \partial \Omega\right) \rightarrow \infty
$$

and $\tilde{u}$ is a nontrivial solution of the limiting problem $\left(\wp_{\infty}\right)$ in $\mathbb{R}^{N}$. Moreover, since $\varepsilon_{k}^{-1}\left|\xi_{k}-y_{k}\right|<C<\infty$ for all $k$, it follows that $y_{k} \in \Omega$.

(4) We define $v_{k} \in H_{0}^{1}(\Omega)^{\tau}$ as follows: let $\varphi \in C^{\infty}\left(\mathbb{R}^{N}\right)$ be radially symmetric and such that $0 \leqslant \varphi \leqslant 1, \varphi \equiv 1$ on $B(0,1)$ and $\varphi \equiv 0$ outside of $B(0,2)$. Let

$$
4 \rho_{k}= \begin{cases}\min \left\{\operatorname{dist}\left(y_{k}, \partial \Omega\right),\left|y_{k}-\tau y_{k}\right|\right\} & \text { in case }(I), \\ \operatorname{dist}\left(y_{k}, \partial \Omega\right) & \text { in case }(I I) .\end{cases}
$$

Thus, $\varepsilon_{k}^{-1} \rho_{k} \rightarrow \infty$. In case $(I)$ we take

$w_{k}=\varepsilon_{k}^{(2-N) / 2}\left[\tilde{u}\left(\varepsilon_{k}^{-1}\left(\cdot-y_{k}\right)\right) \varphi\left(\rho_{k}^{-1}\left(\cdot-y_{k}\right)\right)-\tilde{u}\left(\varepsilon_{k}^{-1} \tau\left(\cdot-\tau y_{k}\right)\right) \varphi\left(\rho_{k}^{-1}\left(\cdot-\tau y_{k}\right)\right)\right]$

and in case $(I I)$

$$
w_{k}=\varepsilon_{k}^{(2-N) / 2} \tilde{u}\left(\varepsilon_{k}^{-1}\left(\cdot-y_{k}\right)\right) \varphi\left(\rho_{k}^{-1}\left(\cdot-y_{k}\right)\right) .
$$

In both cases, $w_{k} \circ \tau=-w_{k}$. We define

$$
v_{k}=u_{k}-w_{k} \in H_{0}^{1}(\Omega)^{\tau} .
$$

As in $[14,17,3]$ one verifies that $v_{k}$ has the desired properties. 
Proof of theorem 7. Since PS-sequences for $E_{\lambda}$ are bounded in $H_{0}^{1}(\Omega)$,

$$
\left\|u_{k}\right\|_{\lambda}^{2}=N E_{\lambda}\left(u_{k}\right)-\frac{N-2}{2} D E_{\lambda}\left(u_{k}\right) u_{k} \rightarrow N c .
$$

Therefore $c \geqslant 0$. We may assume that $u_{k} \rightarrow u$ weakly in $H_{0}^{1}(\Omega)^{\tau}$ and $u_{k} \rightarrow u$ a.e. in $\Omega$. It is easy to see that $D E_{\lambda}(u)=0$ and that $u_{k}^{1}:=u_{k}-u$ is a PS-sequence for $E_{0}$ such that $u_{k}^{1} \in H_{0}^{1}(\Omega)^{\tau}, u_{k}^{1} \rightarrow 0$ weakly in $H_{0}^{1}(\Omega)^{\tau}$ and $E_{0}\left(u_{k}^{1}\right)=E_{\lambda}\left(u_{k}\right)-E_{\lambda}(u)=c-E_{\lambda}(u)+\mathrm{o}(1)$. The result now follows inductively from proposition 16 .

\section{References}

[1] Aubin Th 1976 Problèmes isopérimétriques et espaces de Sobolev J. Diff. Geom. 11 573-98

[2] Brézis H and Nirenberg L 1983 Positive solutions of nonlinear elliptic equations involving critical Sobolev exponents Comm. Pure Appl. Math. 36 437-77

[3] Clapp M A global compactness result for elliptic problems with critical nonlinearity on symmetric domains (Proc. of the Workshop in Nonlinear Differential Equations, Bergamo 2001) Prog. Nonlinear Diff. Eqns Appl. at press

[4] Clapp M and Puppe D 1991 Critical point theory with symmetries J. Reine Angew. Math. 418 1-29

[5] Cerami G, Solimini S and Struwe M 1986 Some existence results for superlinear elliptic boundary value problems involving critical exponents J. Funct. Anal. 69 289-306

[6] Tom Dieck T 1987 Transformation groups de Gruyter Studies in Math vol 8 (Berlin-New York: de Gruyter)

[7] Ekeland I 1974 On the variational principle J. Math. Anal. Appl. 47 324-53

[8] Fortunato D and Jannelli E 1987 Infinitely many solutions for some nonlinear elliptic problems in symmetrical domains Proc. R. Soc. Edinburgh A 105 205-13

[9] Hebey E and Vaugon M 1994 Existence and multiplicity of nodal solutions for nonlinear elliptic equations with critical Sobolev growth J. Funct. Anal. $119298-318$

[10] Lazzo M 1992 Solutions positives multiples pour une équation elliptique non linéaire avec l'exposant critique de Sobolev C.R. Acad. Sci. Paris (Série I) 314 61-4

[11] Pohožaev S 1965 Eigenfunctions of the equation $\Delta u+\lambda f(u)=0$ Sov. Math. Dokl. 6 1408-11

[12] Rey O 1989 A multiplicity result for a variational problem with lack of compactness Nonlinear Anal. TMA 133 1241-9

[13] Struwe M 1984 A global compactness result for elliptic boundary value problems involving limiting nonlinearities Math. Z. 187 511-17

[14] Struwe M 1990 Variational Methods. Applications to Nonlinear Partial Differential Equations and Hamiltonian Systems (Berlin-Heidelberg: Springer)

[15] Talenti G 1976 Best constant in Sobolev inequality Ann. Mat. Pure Appl. 110 353-72

[16] Tarantello G 1992 Nodal solutions of semilinear elliptic equations with critical exponent Diff. Integral Eqns 5 25-42

[17] Willem M 1996 Minimax Theorems PNLDE 24 (Basel: Birkhäuser)

[18] Zhang D 1989 On multiple solutions of $\Delta u+\lambda u+|u|^{4 /(n-2)}=0$ Nonlinear Anal. TMA 13 353-72 